\title{
Relation between the ring current and the tail current during magnetic storms
}

\author{
V. V. Kalegaev ${ }^{1}$, N. Y. Ganushkina ${ }^{2}$, T. I. Pulkkinen ${ }^{2}$, M. V. Kubyshkina ${ }^{3}$, H. J. Singer ${ }^{4}$, and C. T. Russell \\ ${ }^{1}$ Skobeltsyn Institute of Nuclear Physics, Moscow State University, Moscow 119992, Russia \\ ${ }^{2}$ Geophysical Research, Finnish Meteorological Institute, POBox 503, Helsinki, FIN-00101, Finland \\ ${ }^{3}$ Institute of Physics, University of St-Petersburg, St-Petersburg, 198904, Russia \\ ${ }^{4}$ H. J. Singer, NOAA Space Environment Center, Boulder, CO 80305-3328, USA \\ ${ }^{5}$ Institute of Geophysics and Planetary Physics, University of California, Los Angeles, CA 90095-1567, USA
}

Received: 2 March 2004 - Revised: 5 November 2004 - Accepted: 15 November 2004 - Published: 28 February 2005

\begin{abstract}
We study the dynamics of the magnetospheric large-scale current systems during storms by using three different magnetospheric magnetic field models: the paraboloid, event-oriented, and Tsyganenko T01 models. We have modelled two storm events, one moderate storm on 25-26 June 1998, when $D_{s t}$ reached $-120 \mathrm{nT}$ and one intense storm on 21-23 October 1999, when $D_{s t}$ dropped to $-250 \mathrm{nT}$. We compare the observed magnetic field from GOES 8, GOES 9, and GOES 10, Polar and Geotail satellites with the magnetic field given by the three models to estimate their reliability. All models demonstrated quite good agreement with observations. Since it is difficult to measure exactly the relative contributions from different current systems to the $D_{s t}$ index, we compute the contributions from ring, tail and magnetopause currents given by the three magnetic field models. We discuss the dependence of the obtained contributions to the $D_{s t}$ index in relation to the methods used in constructing the models. All models show a significant tail current contribution to the $D_{s t}$ index, comparable to the ring current contribution during moderate storms. The ring current becomes the major $D_{s t}$ source during intense storms.
\end{abstract}

Key words. Magnetospheric physics (Current systems; Magnetospheric configuration and dynamics; Storms and substorms)

\section{Introduction}

Despite the many investigations of storm dynamics made during the recent years, the measure of storm intensity, the $D_{s t}$ index, and the relative contributions to it from different current systems during a storm are still under discussion. The $D_{s t}$ index was thought to be well correlated with the inner ring current energy density from storm maximum well into recovery (Hamilton et al., 1998; Greenspan and Hamilton, 2000). Several studies, however, have suggested that the $D_{s t}$

Correspondence to: V. V. Kalegaev

(klg@dec1.sinp.msu.ru) index contains contributions from many sources other than the azimuthally symmetric ring current (Campbell, 1973; Arykov and Maltsev, 1993; Maltsev et al., 1996; Alexeev et al., 1996; Kalegaev et al., 1998; Dremukhina et al., 1999; Greenspan and Hamilton, 2000; Turner et al., 2000; Alexeev et al., 2001; Ohtani et al., 2001; Liemohn et al., 2001; Ganushkina et al., 2002, 2004; Tsyganenko et al., 2003).

Experimental investigations of the $D_{s t}$ problem are often based on Dessler-Parker-Scopke relation (Dessler and Parker, 1959; Scopke, 1966)

$$
b_{r}=-\frac{2}{3} B_{0} \frac{\varepsilon_{r}}{\varepsilon_{d}},
$$

which relates the magnetic field of the ring current at the Earth's center, $b_{r}$, with the total energy of the ring current particles, $\varepsilon_{r}$, where $\varepsilon_{d}=\frac{1}{3} B_{0} M_{E}$ is the energy of the geomagnetic dipole above the Earth's surface, $B_{0}$ is the geodipole magnetic field at the equator.

The ring current contribution to $D_{s t}$ was studied by Greenspan and Hamilton (2000) based on AMPTE/CCE ring current particle measurements in the equatorial plane for 80 magnetic storms from 1984 until 1989. It was shown that the ring current magnetic field obtained from the total ring current energy using the Dessler-Parker-Scopke relation represents well $D_{s t}$ (especially on the nightside). However, the currents other than the ring current can produce significant magnetic perturbations of different signs at the Earth's surface, so their total magnetic perturbation will be about zero.

The tail current contribution to $D_{s t}$ (to the $S Y M-H$ index, more exactly) was studied by Ohtani et al. (2001) for the 25-26 June 1998 magnetic storm. Based on GOES 8 measurements and their correlation with $D_{s t}$, the authors determined the contribution from the tail current at $D_{s t}$ minimum to be at least $25 \%$. It was established that $D_{s t}$ lost $25 \%$ of its value after substorm onset due to tail current disruption. The question about the preintensification level of tail current magnetic field, which continues to contribute to $D_{s t}$ after substorm dipolarization, remains open. 

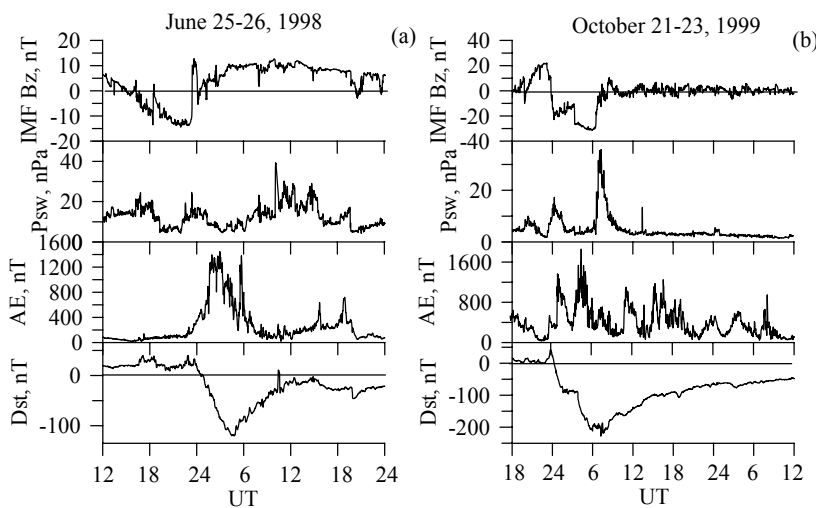

Fig. 1. Overview of 25-26 June 1998 moderate and 21-23 October 1999 intense storm events.

Thus, based only on the measurements, we cannot explicitly distinguish between the contributions from different magnetospheric current systems which contribute to the ground magnetic field. However, we can estimate them by using modern magnetospheric models, which can provide separate calculations of the magnetic field of the different magnetospheric magnetic field sources. Magnetic field modelling is a useful tool for studying the evolution of large-scale current systems during magnetic storms.

The empirical models developed by Tsyganenko (for example, T96 (Tsyganenko, 1995) and earlier versions) are constructed by minimizing the RMS deviation from the large magnetospheric database (Fairfield et al., 1994), which contains magnetospheric magnetic field measurements accumulated over many years. As magnetic storms are relatively rare events during the observation period, their influence on the model coefficients is small. The applicability of the T96 model is limited to $20>D_{s t}>-100 \mathrm{nT}$, $0.5 \mathrm{nPa}<P_{s w}<10 \mathrm{nPa},-10 \mathrm{nT}<B_{z} \mathrm{IMF}<10 \mathrm{nT}$. The version T01 (Tsyganenko, 2002a,b) was developed using a larger database which also includes measurements made in recent years. It is valid over a wider range of parameter values.

The existing theoretical models determine the magnetospheric magnetic field from physical constraints. The paraboloid model of the Earth's magnetosphere (Alexeev, 1978; Alexeev et al., 1996; Alexeev et al., 2001) is based on an analytical solution of the Laplace equations for each largescale current system in the magnetosphere with a fixed shape (paraboloid of revolution). The paraboloid model takes parameters of magnetospheric current systems (intensities and locations) as input. These input parameters are determined from empirical data using submodels. Such a feature allows for easy changes to the paraboloid model parameterization.

Several types of studies require an accurate representation of the magnetospheric configuration during a specific event. For such cases, event-oriented modelling is of key importance (Ganushkina et al., 2002, 2004). Event-oriented models contain free parameters whose values are evaluated from observations for each time period separately.
The main focus of this paper is the relation between the ring current and the tail current during storm times. To study this we use three different magnetic field models: the paraboloid model (Alexeev, 1978; Alexeev et al., 2001), the event-oriented model (Ganushkina et al., 2002), and the T01 model (Tsyganenko, 2002a,b). To investigate the tail current/ring current relationship we model two storm events, one moderate storm on 25-26 June 1998, when $D_{s t}$ reached $-120 \mathrm{nT}$ and one intense storm on 21-23 October 1999, in which $D_{s t}$ dropped to $-250 \mathrm{nT}$. Comparison of the magnetic field given by different models with satellite data allows us to verify the different modelling approaches and their reliability for magnetospheric studies during disturbed conditions. We compute the relative contributions from the ring, magnetotail and magnetopause currents to the $D_{s t}$ index using all three models. Long periods of modelling for each storm allow us to examine and compare the long-term evolution of different current systems during storms with different intensity given by models based on the different approaches.

\section{Description of storm events}

Figure 1 represents the overview of the measurements during the magnetic storms on 25-26 June 1998 and 21-23 October 1999. The solar wind data and IMF were obtained from Wind spacecraft, taking into account the convection time shift of about 40 min.

On 25 June 1998 the IMF $B_{z}$ behavior (Fig. 1a) reflected the passage of a magnetic cloud: southward turn at 15:50 UT when $B_{z}$ reached $-13 \mathrm{nT}$ and then suddenly jumped to more than $+15 \mathrm{nT}$ around 23:00 UT. At 24:00 UT $B_{z}$ decreased rapidly to $-5 \mathrm{nT}$ and began a new slower enhancement to the level of about $10 \mathrm{nT}$ which is approached at 05:00 UT on 26 June. The solar wind dynamic pressure had several peaks around $20-30 \mathrm{nPa}$. The $A E$ index showed the first increase at about 23:00 UT on 25 June but the maximum substorm activity was detected during 02:00-04:00 UT on 26 June with a peak value of 14:00 nT around 02:55 UT. The $D_{s t}$ index started to decrease at the beginning of 26 June and reached $-120 \mathrm{nT}$ around 05:00 UT, six hours later the first northward $B_{z}$ reversal occurred, after a long period of substorm activity when IMF $B_{z}$ demonstrated relatively slow growth from $-5 \mathrm{nT}$ to $+10 \mathrm{nT}$. The detailed analysis and interpretation of this interesting phenomena was made by Ohtani et al. (2001).

Figure $1 \mathrm{~b}$ shows an overview of the intense storm on 2123 October 1999. IMF $B_{z}$ turned from $+20 \mathrm{nT}$ to $-20 \mathrm{nT}$ at about 23:50 UT on 21 October and after some increase during the next three hours dropped down to $-30 \mathrm{nT}$ around 06:00 UT on 22 October. After that, the IMF $B_{z}$ oscillated around zero. Solar wind dynamic pressure showed two main peaks, a $15 \mathrm{nPa}$ peak around 24:00 UT on 21 October and a $35 \mathrm{nPa}$ peak around 07:00 UT on 22 October. There were several peaks in the $A E$ index reaching $800-1600 \mathrm{nT}$. The $D_{s t}$ index dropped to $-230 \mathrm{nT}$ at 06:00-07:00 UT on $22 \mathrm{Oc}-$ tober. 


\section{Storm-time magnetic field models}

\subsection{Paraboloid model}

The basic equations of the paraboloid model represent the magnetic fields of the ring current, of the tail current including the closure currents on the magnetopause, of the Region 1 field-aligned currents, of the magnetopause currents screening the dipole field and of the magnetopause currents screening the ring current (Alexeev, 1978; Alexeev et al., 1996; Alexeev et al., 2001). Here we discuss the latest version of the model, A2000 (Alexeev et al., 2001). In the A2000 model (as in the previous versions of paraboloid model) the magnetopause is set to be a paraboloid of revolution. The condition $B_{n}=0$ is assumed at the magnetopause. The model parameters determining the large-scale magnetospheric current systems are the following: the geomagnetic dipole tilt angle $\psi$, the magnetopause stand-off distance $R_{1}$, the distance to the inner edge of the tail current sheet $R_{2}$, the magnetic flux through the tail lobes $\Phi_{\infty}$, the ring current magnetic field at the Earth's center $b_{r}$, and the maximum intensity of the field-aligned current $I_{\|}$. At each moment the parameters of the magnetospheric current systems define the instantaneous state of the magnetosphere and can be determined from observations.

The A2000 model parameterization is described in detail by Alexeev et al. (2001). The geocentric distance $R_{1}$ to the subsolar point is calculated using solar wind data: solar wind dynamical pressure and IMF $B_{z}$ component (Shue et al., 1997). The distance to the inner edge of the tail current sheet $R_{2}$ is obtained by mapping the equatorward boundary of the auroral oval at midnight, $\varphi_{n}=74.9^{\circ}-8.6 \log _{10}\left(-D_{s t}\right)$, as given by Starkov (1993), to the equatorial plane. The magnetic flux across the tail lobe is a sum of two terms $\Phi_{\infty}=\Phi_{0}+\Phi_{s}$, which depend on the tail current density, $R_{1}$ and $R_{2}$. The first term corresponds to a slow adiabatic evolution of the tail current due to solar wind variations and remains constant $\left(\Phi_{0}=3.7 \cdot 10^{8} \mathrm{~Wb}\right)$ while the second term $\Phi_{s}=\frac{-A L}{7} \frac{\pi R_{1}^{2}}{2} \sqrt{\frac{2 R_{2}}{R_{1}}+1}$ is associated with substorms. Here $\Phi_{s}$ variations represent the integrated substorm activity dependent on the hourly-averaged AL-index (see Alexeev et al., 2001).

According to Burton et al. (1975) and the Dessler-ParkerSckopke relation (1) the ring current magnetic field variation at the Earth's center is given by $\frac{d b_{r}}{d t}=F(E)-\frac{b_{r}}{\tau}$, where $F(E)$ is the injection function defined in accordance with Burton et al. (1975); O'Brien and McPherron, (2000), and $\tau$ is the lifetime of the ring current particles. Burton et al. (1975) and O'Brien and McPherron (2000) found the average values of the amplitude of the injection function ( $d$ in notation of (Burton et al., 1975; O'Brien and McPherron, 2000)), but apparently it varies from storm to storm. In Alexeev et al. (2001) $d$ was obtained from independent research by Jordanova et al. (1999). In these case studies we will find $d$ which provides the minimum RMS deviation between $D_{s t}$ and the modelled $D_{s t}$. In such an approach $b_{r}$ will include not only a contribution from the symmetrical ring current but also the symmetrical magnetic fields from the other magnetospheric magnetic field sources, which are not included in A2000. First of all, this is the symmetrical part of the partial ring current magnetic field.

$I_{\|}$is determined from the IMF $B_{z}$ component, and solar wind velocity and density as described by Alexeev and Feldstein (2001).

As a result the A2000 allows one to calculate the magnetic field depending on the described above parameters of magnetospheric current systems, which can be obtained from input data: date, IMF, solar wind density and velocity, $A L$ and $D_{s t}$ indices.

\subsection{Event-oriented model by Ganushkina et al.}

The Ganushkina et al. (2002, 2004) storm-time magnetic field model (G2003) used the Tsyganenko T89 magnetic field model (Tsyganenko, 1989) as a baseline, and the ring, tail and magnetopause currents were modified to give a good fit with in-situ observations.

The ring current model consists of symmetric and asymmetric parts (Ganushkina et al., 2004) represented by a Gaussian distribution of the current density. The total current density of the symmetric ring current is a sum of eastward and westward current intensities. The asymmetric partial ring current is closed by field-aligned currents flowing from the ionosphere at dawn and into the ionosphere at dusk, in the Region 2 current sense. The magnetic field from this current system is calculated using the Biot-Savart law. For the tail current system both global intensification of the tail current sheet and local changes in a thin current sheet were implemented (Ganushkina et al., 2004). To adjust for the magnetopause inward motion during increased solar wind dynamic pressure, the magnetic field of the Chapman-Ferraro currents $B_{C F_{\mathrm{T} 89}}$ at the magnetopause was scaled using the solar wind dynamic pressure.

The free parameters in the model are the radial distance of the westward ring current $\left(R_{0 \text { west }}\right)$ and partial ring current $\left(R_{0 \text { part }}\right)$, and the maximum current densities for westward $\left(J_{0 \text { west }}\right)$ and partial $\left(J_{0 \text { part }}\right)$ ring currents, the amplification factor for the tail current $(A T S)$, and the additional thin current sheet intensity $\left(A_{\text {ntc }}\right)$. By varying the free parameters we found the set of parameters that gives the best fit between the model and the in-situ magnetic field observations. The details of the fitting procedure can be found in Ganushkina et al. (2002).

\subsection{Tsyganenko T01 model}

In the T01 model (Tsyganenko, 2002a,b) the general approach is to parameterize the current systems and evaluate these parameter values in a statistical sense, using a large magnetospheric database. Several revisions were introduced in the mathematical description of the major sources of the magnetospheric field and in their parameterization with respect to the earlier T96 model (Tsyganenko, 1995). A partial 

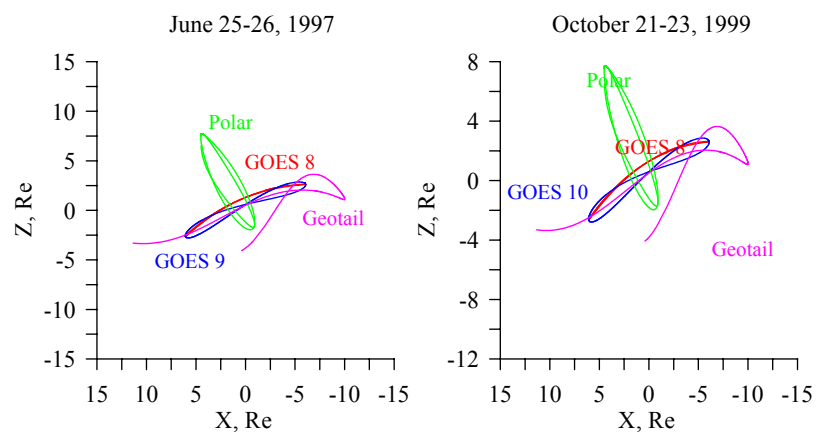

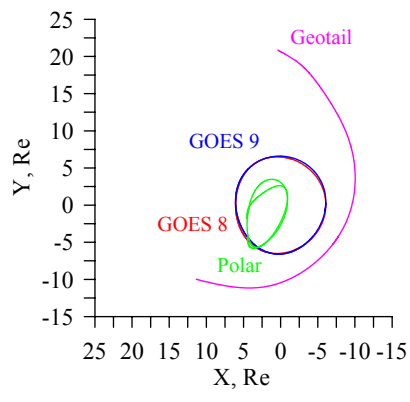

(a)

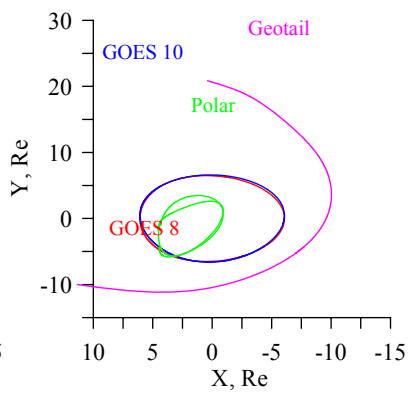

(b)
Fig. 2. Evolution of orbits of satellites during the time periods when the magnetic field data was used for modelling storm events on (a) 25-26 June 1997, and (b) 21-23 October 1999.

ring current with field-aligned closure currents are included, and the cross-tail current sheet is warped in two dimensions in response to the geodipole tilt, with its inner edge shifting along the Sun-Earth line and its thickness varying along and across the tail. The magnetopause is specified according to the empirical model by Shue et al. (1997).

The model parameters are geodipole tilt angle, IMF $B_{y}$ and $B_{z}$ components, solar wind dynamic pressure, and $D_{s t^{-}}$ index. An attempt is made to take into account the prehistory of the solar wind by introducing two functions, $G_{1}$ and $G_{2}$, that depend on the IMF $B_{z}$ and solar wind velocity and their time history.

\section{Comparison of modelling results: magnetic field}

To contrast and to examine the reliability of the three models, we present here a comparison of the model results with magnetic measurements from various spacecraft during the June 1998 and October 1999 storms. We calculate the magnetic field along the spacecraft orbits located in the different regions of space: geostationary orbit (GOES $-8,-9$, and -10), near-Earth's tail (Geotail), and high-latitude magnetosphere (Polar). Analysis of simultaneous measurements in the different magnetospheric regions helps to determine the role of different magnetospheric current systems during magnetic storms.

Figure 2 shows the evolution of orbits in the noonmidnight meridional (upper panels) and equatorial (lower
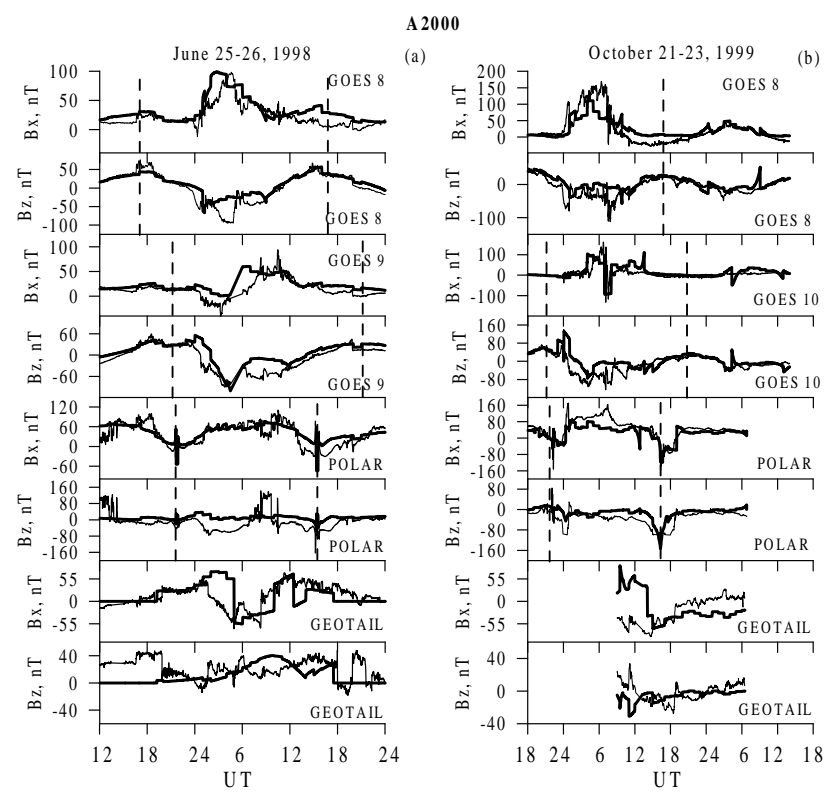

Fig. 3. Comparison of the observed $B_{x}$ and $B_{z}$ components of the external magnetic field in the GSM coordinates (thin lines) with A2000 model results (thick lines) for GOES 8 (two upper panels), GOES 9 and GOES 10 (next two panels), Polar (next two panels) and Geotail (bottom two panels) for (a) 25-26 June 1998 and (b) for 21-23 October 1999 storm events.

panels) planes of satellites such as GOES 8 (red curve), GOES 9 or 10 (blue curve), Polar (green curve), and Geotail (pink curve), during the time periods when the magnetic field data were used for modelling storm events on (a) 25-26 June 1997, and (b) 21-23 October 1999. All measurements were made inside the magnetosphere.

Figure 3 shows the $B_{x}$ and $B_{z}$ components of the external magnetic field obtained from observations shown by thin lines and A2000 model results shown by thick lines for GOES 8 (two upper panels), GOES 9 and GOES 10 (next two panels), Polar (next two panels) and Geotail (bottom two panels) for (a) 25-26 June 1998 and (b) for 21-23 October 1999 storm events. Dashed grid lines show the noon locations for GOES spacecraft, and perigees of the Polar orbit. Figures 4 and 5 show the observed and model magnetic fields in the same format for the event-oriented model G2003 and the Tsyganenko T01 model, respectively. $B_{x}$ and $B_{z}$ measured components represent the main changes in the magnetospheric current systems. Their comparisons with the model results reveal the main model's features.

It can be seen that generally all models show quite good agreement with observations. For the moderate storm the $B_{x}$ measured at geosynchronous orbit is better represented by the A2000 and T01 models, whereas the G2003 model gives a more accurate reproduction of the $B_{z}$ component. The large observed $B_{x}$ values imply the existence of intense currents that can be either field-aligned or perpendicular, or an even stronger compression of the magnetosphere than that represented by the magnetopause current intensification in 
Table 1. The RMS deviations in $\mathrm{nT}$ between the observed and modelled magnetic field calculated by the paraboloid (A2000, Alexeev et al., 2001), event-oriented (G2003, Ganushkina et al., 2003), and Tsyganenko (T01, Tsyganenko, 2002a,b) models during magnetic storms on 25-26 June 1998 and 21-23 October 1999.

\begin{tabular}{lrrr}
\hline Satellites & A2000 & G2003 & T01 \\
\hline 25-26 June 1998 & & & \\
GOES 8 & 18.9 & 16.8 & 18.3 \\
GOES 9 & 21.2 & 22.4 & 16.5 \\
Polar & 26.7 & 33.5 & 28.2 \\
Geotail & 28.4 & 21.0 & 21.6 \\
21-23 October 1999 & & & \\
GOES 8 & 37.0 & 30.2 & 32.1 \\
GOES 10 & 33.7 & 29.4 & 32.7 \\
Polar & 40.0 & 35.4 & 32.7 \\
Geotail & 22.6 & 11.8 & 11.4 \\
& & & \\
\hline
\end{tabular}

the G2003 model. The A2000 model represents the magnetopause size variations, depending not only on solar wind pressure but also on IMF $B_{z}$ based on Shue et al. (1997) model. The A2000 describes the $B_{x}$ values during the magnetic storm main phase (the first $6 \mathrm{~h}$ of 26 June 1998) more accurately than the other models. On the other hand, the A2000 model underestimates the $B_{z}$ values during this time interval. This is because the paraboloid model represents the cross-tail currents as a discontinuity between the oppositely directed magnetic field bundles in the southern and northern tail lobes and as a result gives a very small $B_{z}$ component in the vicinity of the tail current.

In general, all three models show approximately similar accuracy in the representation of magnetic field data observed by Polar. The G2003 model magnetic field agrees with the observed field at Geotail (from 00:20 UT, 25 June until 18:00 UT 26 June while the spacecraft was inside the magnetosphere) slightly better than that given by the A2000 and T01 models.

During the intense storm on 21-23 October 1999 the $B_{x}$ components from GOES 8 and GOES 10, and Polar, are best represented by the T01 model. At the same time, the T01 model underestimates the $B_{z}$ component significantly at the storm maximum. Model $B_{z}$ values were equal to $-230 \mathrm{nT}$ and $-250 \mathrm{nT}$ around 06:00 UT on 22 October 1999 while the observed ones were $-50 \mathrm{nT}$ and $-80 \mathrm{nT}$ at GOES 8 and GOES 10, respectively. At that time, GOES 8 was around midnight and GOES 10 was moving toward midnight in the dusk sector. At the storm maximum, Polar observations on the duskside showed $B_{z}=-25 \mathrm{nT}$ while the T01 model gave $B_{z}=-100 \mathrm{nT}$. Similarly to the moderate storm, the G2003 model reproduces the $B_{z}$ variations at GOES and Polar with enough accuracy.

The local magnetic field variations near the magnetospheric tail current sheet along the Geotail orbit are not quite correctly reproduced by the models. The A2000 model gives

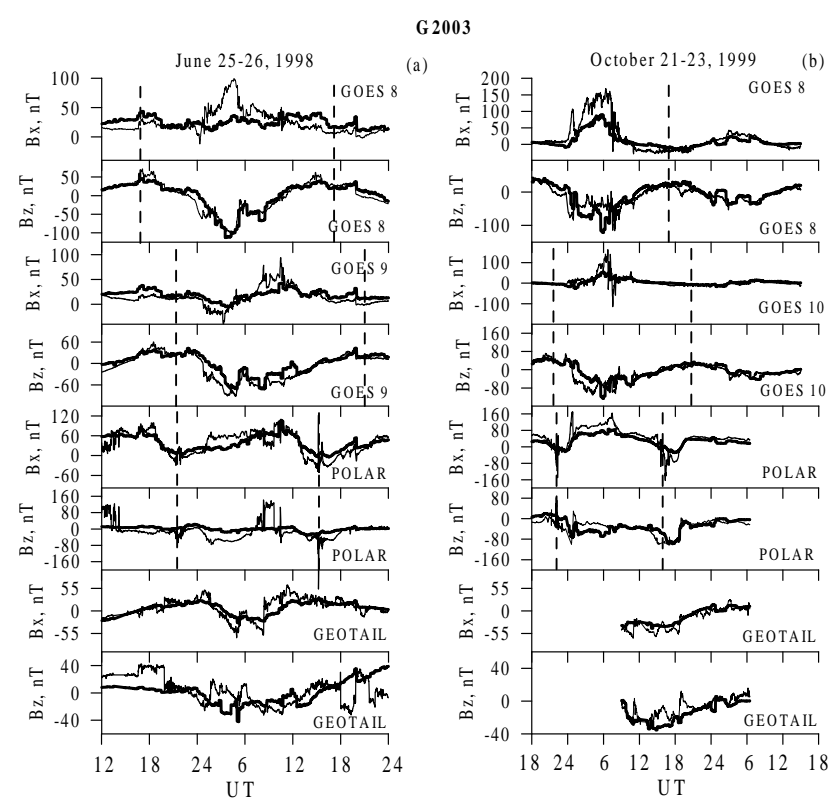

Fig. 4. Observed and model magnetic fields in the same format as in Fig. 3 for the event-oriented model G2003.
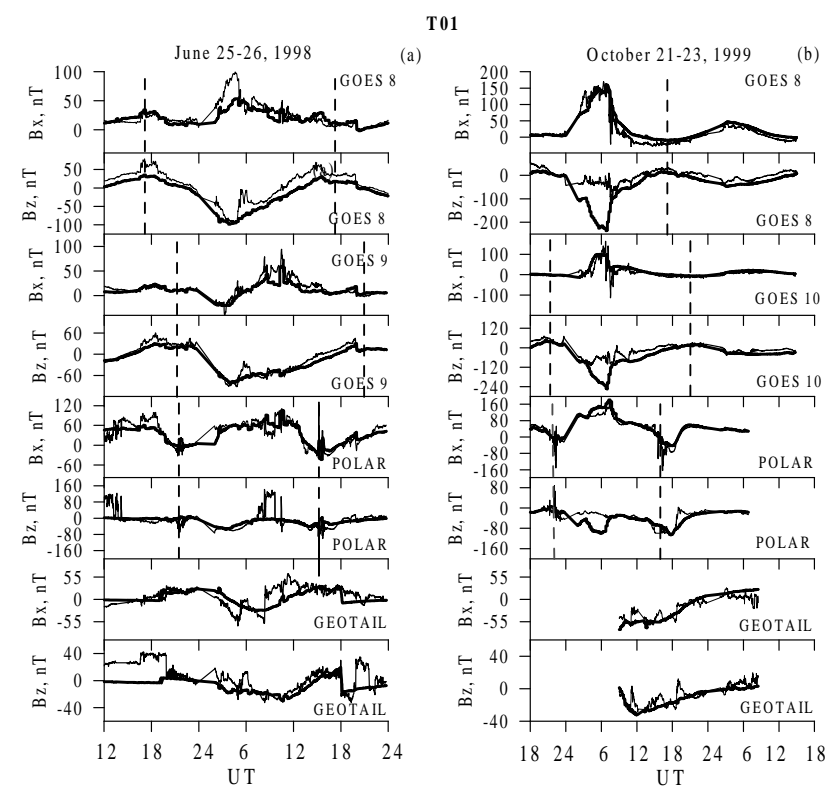

Fig. 5. Observed and model magnetic fields in the same format as in Fig. 3 for the the empirical T01 model.

additional discrepancies (e.g. $B_{x}$ drops) that arise from the construction of the tail current model discussed above. However, for both storm events the $B_{x}$ components are described with a reasonable accuracy at GOES 8 and GOES 10, as well as at Polar.

Table 1 shows the RMS deviations between the satellite measurements and model calculations determined as $\delta B=\sqrt{\frac{1}{N} \sum_{i=1}^{N}\left(B_{\mathrm{obs}}-B_{\text {model }}\right)^{2}}$. The obtained discrepancies are calculated during the whole considered time-intervals 
and include quiet as well as disturbed periods. We note that for each orbit the models give the accuracy of about half of the average value of the magnetic field. In general, all models represent well the global variations of magnetospheric magnetic field measured by spacecraft. However, the model features determine the specific behavior of the magnetic field calculated in different magnetospheric regions by different models during the different phases of the considered magnetic storms.

The paraboloid model reproduces well the $B_{x}$ components of the magnetic field measured along the GOES and Polar orbits for any level of disturbances but underestimates the $B_{z}$ depression, due to tail current model features and possibly due to the absence of the partial ring current model in A2000. The T01 model also provides good agreement between the observed and modelled $B_{x}$ component. On the other hand, during the intense storm maximum, the model $B_{z}$ is significantly more depressed than that observed along the GOES and Polar orbit. Because the ring current cannot give the significant contribution to the magnetic field at geostationary orbit, we propose that this discrepancy is due to an overestimation of the tail current contribution. Apparently, this is the consequence of the general approach used in development of any empirical model. Calculation results are very sensitive to the database used for the model construction. Intense storms are only a small part of such databases. As a result just during extremely disturbed conditions the empirical model demonstrates the sufficient discrepancies. The event-oriented model G2003 represents better the substorm-associated variations of the $B_{z}$ component at geosynchronous orbit during both moderate and intense storms, but gives discrepancies in the $B_{x}$ variation during storm maximum.

\section{Comparison of modelling results: $D_{s t}$ index}

\subsection{Model calculations of $D_{s t}$ index}

In this study, along with Alexeev et al. (2001), we suggest that the magnetopause, tail and ring currents are the main contributors to the $D_{s t}$ index. Although the models considered above are also able to calculate the magnetic field from the other magnetospheric currents (see Sect. 3), their contributions to $D_{s t}$ are not addressed in this study.

The storm-time magnetic field depression at the Earth's surface is determined mainly by ring current, tail current and partial ring current. However, their relative strength and location in the inner magnetosphere remains ambiguous, and it is difficult to separate in the measurements the partial ring current from the storm-time tail and symmetrical ring currents. Obviously, the magnetic field of the partial ring current has a symmetrical part which contributes to the $D_{s t}$-index. The different estimates for the effect of the partial ring current on $D_{s t}$ were obtained by Liemohn et al. (2001), as the dominant contribution during the magnetic storm main phase, and by Tsyganenko et al. (2003), as about 1/7 of the total ring current contribution during storm maximum. Because the question about the partial ring current contribution to $D_{s t}$ requires special consideration, it will not be the subject of this paper. Along with Ganushkina et al. (2002), we propose in our calculations that the partial ring current produces a part of the total ring current magnetic field variation measured at the Earth's surface. Actually, it is included in the ring current magnetic field calculated in terms of the G2003 and T01 models.

Moreover, the partial ring current is not included in the A2000 model. Possibly, this is the reason for the discrepancies found during comparison between the model calculations and data measured along the spacecraft orbits. However, the symmetrical part of its magnetic field is included in the ring current magnetic field in terms of the approach used for $b_{r}$ calculation (see Sect. 3.1). So, A2000 allows one to calculate the total symmetrical ring current magnetic field (originated from both symmetrical and partial ring current) as well as the total ring current contribution to $D_{s t}$

Earlier studies have given different relative contributions from the magnetospheric current systems to the $D_{s t}$ index. These differences can be very large: the tail current contribution to $D_{s t}$ was $\sim 25 \%$ in a study by Turner et al. (2000) while the tail current contribution was comparable to the $D_{s t}$ in Alexeev et al. (2001) for the same event on 9-12 January 1997. In the present paper we calculate the magnetopause, ring and tail currents storm-time variations at the Earth's surface. The contribution of the ground induced currents to the measured perturbation field is assumed to be $30 \%$ of the magnetic perturbation at the Earth's surface (Häkkinen et al., 2002). The magnetic field horizontal components $(\Delta H(t))$ were computed from the external current systems at the locations of six near-equatorial stations (geomagnetic latitude and longitude are in brackets): Sun Juan $\left(29.9^{\circ}, 8.2^{\circ}\right)$, Tenerife $\left(19.8^{\circ}, 61.4^{\circ}\right)$, Tbilisi $\left(36.8^{\circ}, 116.6^{\circ}\right)$, Lunping $\left(17.6^{\circ}\right.$, $\left.192.0^{\circ}\right)$, Kakioka $\left(28.3^{\circ}, 210.8^{\circ}\right)$, Honolulu $\left(21.8^{\circ}, 268.7^{\circ}\right)$ and Del Rio $(39.0,324.1)$. Then, the quietest day of the month was determined using the World Data Center catalogue, and the magnetic field variation during this quiet day, $\Delta H_{q}(t)$, was calculated from the model. The model $D_{s t}(S Y M-H)$ is then

$\operatorname{Dst}(t)=\frac{1}{N} \sum_{i=1}^{N} \frac{\Delta H_{i}(t)-\Delta H_{q i}(t)}{\cos \theta_{i}}$,

where $N$ is the number of stations (6), and $\theta_{i}$ represents the magnetic latitudes of the stations. This procedure was repeated for total $D_{s t}$ and for contributions from the different current systems. This method of $D_{s t}$ computation is similar to the official procedure described by Sugiura and Kamei (1991). It allows us to unambiguously derive the $D_{s t}$ variations arising from changes in the magnetospheric current systems in the various models.

\subsection{Model $D_{s t}$ index and its sources}

The quiet days for the two storm events were 17 June 1998 and 20 October 1999 for the 25-26 June 1998 and 21-23 

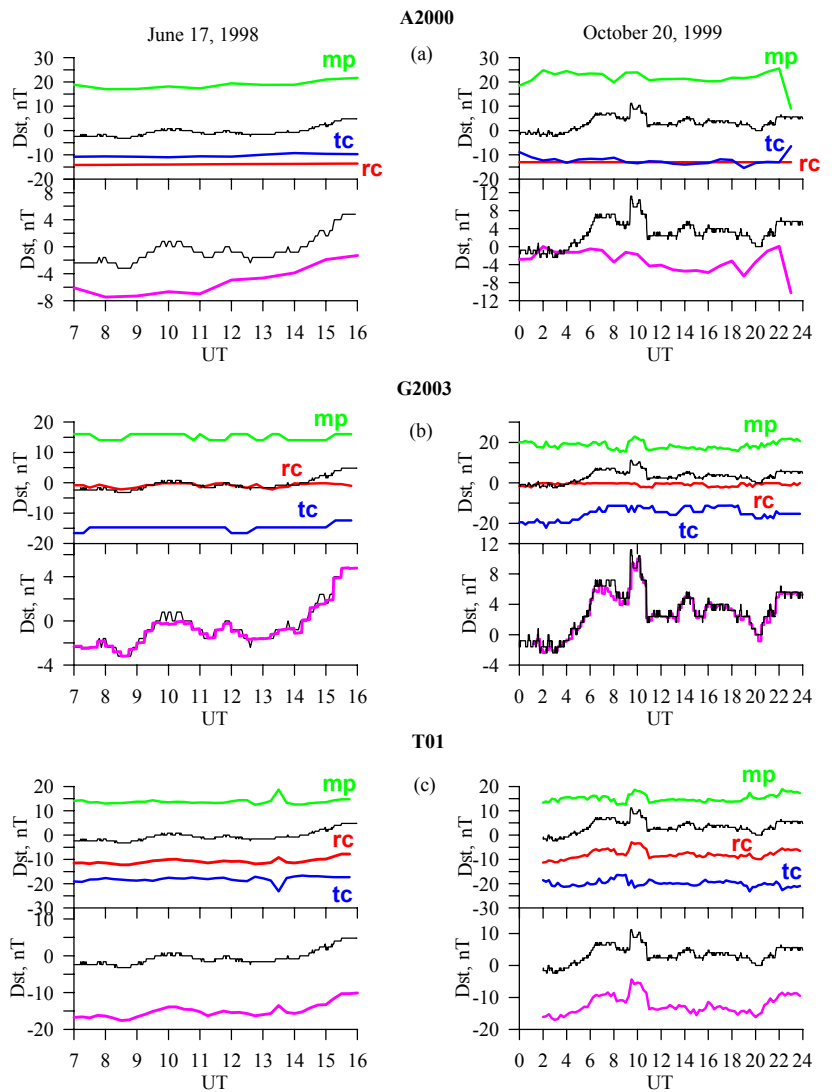

T01

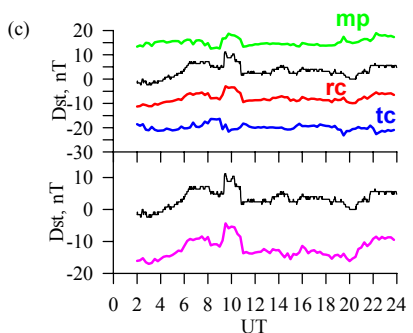

Fig. 6. $D_{s t}$ index (black) and the model contributions to the quiettime magnetic field at the Earth's equator from the magnetopause current (green), ring current (red) and tail current (blue) (top panel) together with the total observed $D_{s t}$ (black) and modelled quietday variation, $\delta H_{q}$, (purple) (bottom panel) for 17 June 1998 (left) and 20 October 1999 (right) using (a) A2000 paraboloid model, (b) G2003 event-oriented model, and (c) T01 model, respectively.

October 1999 storms. The average quiet time fields were $-0.58 \mathrm{nT}$ and $2.74 \mathrm{nT}$, respectively.

Figure 6 shows an analysis of the model current contributions to the quiet-time $D_{s t}$-index for 17 June 1998 (left) and 20 October 1999 (right), using (a) the A2000 paraboloid model, (b) the G2003 event-oriented model, and (c) the T01 model, respectively. The ground-induced currents' effect (30\% of the variation) was taken into account in all the calculations.

We can see that the amplitudes of the calculated variations are about $8-10 \mathrm{nT}$ for all the models (see the bottom panels), but the average values are different. The average quiet day magnetic field variations computed from the A2000 and G2003 models are close to zero. They are about $-5 \mathrm{nT}$ for both events in terms of the A2000 model and about $0 \mathrm{nT}$ and $2.5 \mathrm{nT}$ in terms of the G2003 model. Thus, the magnetic field variation calculated at the Earth's surface by these models during the disturbed conditions can be taken as $D_{s t}$. However, the contributions from the individual current systems to $D_{s t}$ are, of course, not zero. Unlike the A2000 and G2003 models, the T01 model gives a quiet day magnetic field varia-
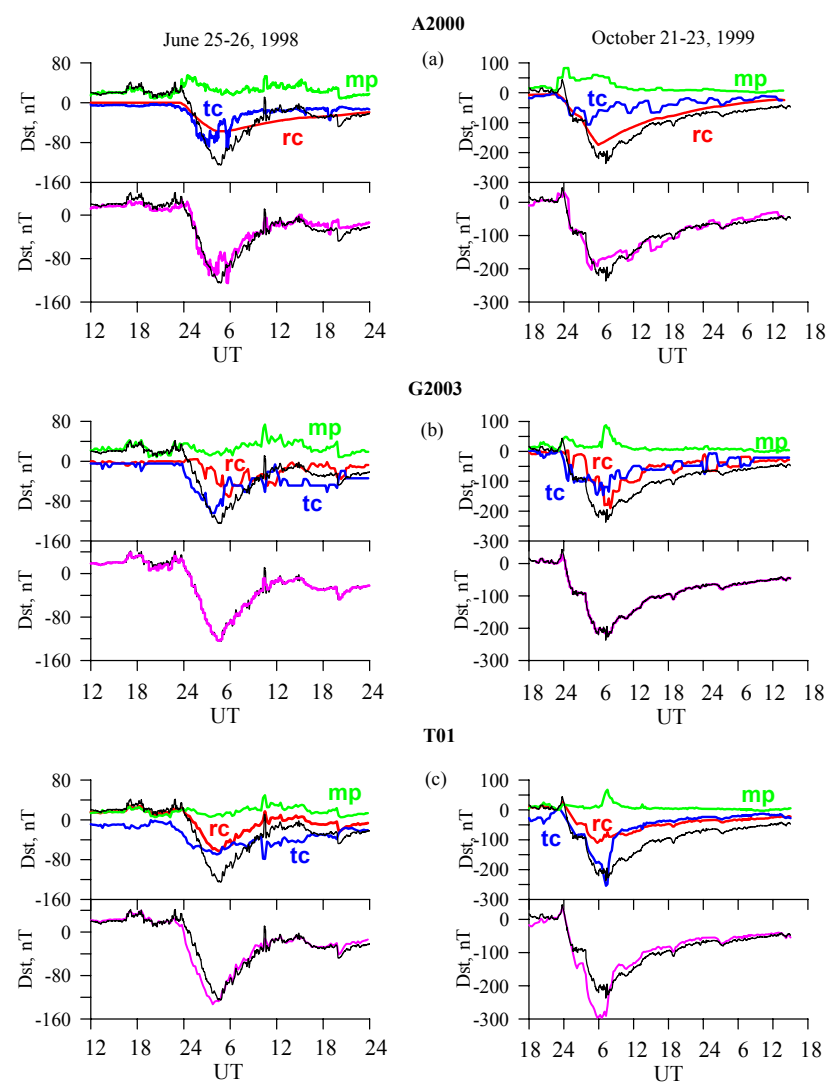

Fig. 7. Model contributions to $D_{s t}$ and total $D_{s t}$ during 25-26 June 1998 and 21-23 October 1999 storm events in the same format as in Fig. 5. The quiet-time contributions from the different current systems are subtracted from the model magnetic field variations.

tion of about $-20 \mathrm{nT}$. Subtracting this value from the ground magnetic field variation during disturbed conditions is an important step in the $D_{s t}$ calculations by the T01 model.

It is important to note that the different quiet-time levels are features of the models and possibly are not connected with the real quiet level magnetic field. In particular, it seems that the large quiet-time field in the T01 model is caused by a relatively small number of measurements in the inner magnetosphere in the database used for T01 construction Tsyganenko et al. (2002a,b). The question about the real quiet time magnetic field level at the Earth's surface remains open for now (see Greenspan and Hamilton (2000).

Figure 7 shows the model contributions and total $D_{s t}$ during 25-26 June 1998 and 21-23 October 1999 storm events in the same format as in Fig. 6 . The quiet time level and quiet time contributions from the different current systems are subtracted from the model magnetic field variations. In general, all three models provide $D_{s t}$, which is in good agreement with the observed $D_{s t}$ index.

During the moderate storm on 25-26 June 1998, the A2000 and G2003 models show that the tail current begins to develop before the ring current and tail current decay begins earlier than that of the ring current. Its contribution to the $D_{s t}$ index almost follows the drop in the total $D_{s t}$. 
The tail current in the T01 model develops even earlier than the $D_{s t}$ starts to decrease. During the storm main phase all models show that the tail and ring current have comparable contributions to the $D_{s t}$. During the recovery phase the ring current remains more enhanced than the tail current according to A2000 and G2003 models, although the G2003 model provides even more tail current contribution than the A2000 model. The ring current in the T01 model recovers rapidly and the tail current remains at an enhanced level almost until the end of the storm recovery.

The situation is quite different during the intense storm on 21-23 October 1999. In all three models the tail current develops first when $D_{s t}$ begins to decrease in a manner similar to the tail current behavior during the moderate storm. During the storm maximum the ring current is the dominant contributor to the $D_{s t}$ index in the A2000 and G2003 models. In the T01 model the tail current continues its development until the storm maximum and gives a major contribution to the $D_{s t}$ index, whereas the ring current contributes only about one third of the tail current contribution. During the recovery phase the tail current contribution decreases and becomes comparable to the ring current contribution.

The tail current contribution to the $D_{s t}$ index computed from the A2000 and G2003 models changes during the magnetic storm. It correlates with substorm activity, and approaches its maximum during substorm maximum estimated by $\mathrm{AE}$ enhancement. On the other hand, the ring current correlates with the total $D_{s t}$, and its maximum tends to be near the $D_{s t}$ maximum. During the moderate storm, the maximum tail and ring current contributions to $D_{s t}$ were about $70 \%$ and $50 \%$ of maximum $D_{s t}$ in the A2000 model, $85 \%$ and $50 \%$ of maximum $D_{s t}$ in the G2003 model, and 50\% and $50 \%$ of maximum $D_{s t}$ in the T01 model. During the intense storm the maximum tail and ring current contributions were, respectively, about $50 \%$ and $90 \%$ for A2000, $70 \%$ and $90 \%$ for G2003, and $100 \%$ and $40 \%$ for T01 (note that the $D_{s t}$ sources reach their maximums at different UTs). Ring current contribution is determined by injection intensity. Amplitude of the injection function $F(E)$ (see Sect. 3.1) calculated in A2000 for the magnetic storm on 21-23 October $1999 d=-3.8 n T / h(m V / m)^{-1}$ exceeds by absolute value $d=-2.8 n T / h(m V / m)^{-1}$ calculated during the 25-26 June 1998 magnetic storm. It looks reasonable to propose that the stronger storm corresponds to the stronger ring current injection and the larger amplitude of injection (by absolute value). However, this conclusion requires more detailed statistical consideration.

In general, all the models confirm the assumption that the tail current magnetic field can be sufficiently large to provide a significant contribution to the $D_{s t}$, variation (Alexeev et al., 1996). However, the global A2000, G2003 and T01 models demonstrate different tail current development during magnetic storms. While during the moderate storm the tail current and ring current have approximately equal maximum contributions to $D_{s t}$ during the strong magnetic storm the models reveal a different behavior. The tail current becomes the major contributor to $D_{s t}$ in the T01 model, while the tail current contribution is smaller than that of the ring current in the A2000 and G2003 models.

The total $D_{s t}$ computed from the T01 model differs significantly from the measured $D_{s t}$ during the main phase of the magnetic storm. Comparison with GOES 8 and GOES 10 data also shows that the model $B_{z}$ is much smaller than the observed one during the 21-23 October 1999 magnetic storm maximum. Because the ring current magnetic field at geosynchronous orbit is relatively small, the source of the discrepancies in $D_{s t}$ and in $B_{z}$ along the GOES orbit is probably caused by the strong intensification of the tail current in the model. The T01 model represents well $D_{s t}$ and spacecraft measurements during moderate magnetic storms, but does not match $D_{s t}$ during intense magnetic storm maximum. This is a known limitation of the empirical models based on the data of satellite measurements. Possibly, the latest Tsyganenko model (Tsyganenko et al., 2003), which is based on the storm-time data, allows one to obtain the more realistic results during strongly disturbed conditions.

The event-oriented G2003 model, which is also based on empirical data, gives excellent results in reproducing $D_{s t}$, as it uses measurements obtained during the magnetic storm which is modelled. This highlights the complexity of the magnetospheric response to the solar wind driving, and the consequent need for event-oriented modelling.

\section{Discussion}

Three magnetospheric models based on very different approaches (theoretical, empirical and event-oriented) were used in our calculations of the magnetic field. The solar wind data and geomagnetic indices are used as input for theoretical A2000 and empirical T01 models, while the entire existing database of the measurements inside the magnetosphere is the base of the G03 model. The models have the different parameterizations, but we used a unified procedure of $D_{s t}$ and $D_{s t}$-source calculations in terms of all the models, corresponding to the official procedure of $D_{s t}$ derivation from data of ground measurements. This procedure includes subtraction of the quietest day effect and takes into account the magnetic field produced by the Earth's induced currents. Such an approach enables unambiguous determination and accurate comparison of the $D_{s t}$ contributions produced by the magnetospheric current systems in terms of the A2000, G2003 and T01 models.

In this paper we are interested in the relation between ring and tail current. We assume that the ring current magnetic field includes a contribution from the symmetrical ring current, as well as the longitudinal averaged part of the partial ring current, magnetic field. In fact, the ring current includes symmetrical and asymmetrical parts in T01 and G2003, while the symmetrical part of the partial ring current is included in the ring current model in A2000. The ring current (including the partial ring current), tail current and magnetopause currents are proposed to be the main contributors to the $D_{s t}$ index. The models of these currents used 
in the A2000, T01 and G2003 models were described in detail in Alexeev et al. (1996; 2001); Tsyganenko (2002a,b); Ganushkina et al. (2002; 2004). They satisfactorily reflect the main features of the observed current systems but have slightly different geometry and depend on different parameters. For example, the tail current system represented by the models consists of cross-tail currents and closure currents on the magnetopause. The different tail current geometry plays a significant role in the magnetic field calculation near the tail current sheet (see the comparison with Geotail measurements, Sect. 4) but hardly influences the magnetic field variations at the Earth's surface. Otherwise, the tail current intensity, as well as the geocentric distance to the tail current inner edge, determine strongly the $D_{s t}$ dynamics during the magnetic storm. During storm maximum the tail current is located close to the Earth and becomes sensitive to the solar wind dynamic pressure, IMF, and flux content of the tail. So therefore, we would expect that the parameters of the tail current, and consequently its effect on the $D_{s t}$ index are controlled by the factors originated from the solar wind and magnetosphere. The dependence of the model parameters on the external factors (e.g. measured solar wind data) determines the model parameterization. We can see from our calculations that the differences in the parameterization of the models provide the main differences between the $D_{s t}$ calculated by the A2000, G2003 and T01 models.

In spite of the different model's parameterizations, the results obtained by all the models show that the tail current plays a significant role in the magnetic storm development. Computations of the tail current contribution to $D_{s t}$ using the A2000, G2003 and T01 models, show that the tail current contribution to $D_{s t}$ can approach values comparable to the ring current contribution to $D_{s t}$ during storm maximum. The calculations show that 1 ) the relationship between tail and ring currents depends on magnetic storm intensity, and 2) this relationship changes during the course of the magnetic storm development.

It was shown that the theoretical A2000 and event-oriented G2003 models give a tail current contribution to $D_{s t}$ comparable with the ring current contribution during a moderate storm, but that the ring current becomes the dominant contributor during an intense storm (see also Ganushkina et al., 2004). Although we did not analyze the substorm related processes, we can conclude that the level of substorm activity influences the value of the tail current contribution to $D_{s t}$. We suggest that the tail current can produce its maximum contribution to $D_{s t}$ for moderate storms while the ring current remains yet undeveloped. During severe storms, the ring current continues to develop while the tail current has already approached its maximum values. In particular, we can see that the hourly $A L$ index can approach approximately the same maximum values during both moderate and intense storms. The magnetic flux through the polar cap, calculated by the paraboloid model (see Sect. 3.1), as well as the polar cap area, depend strongly on the level of substorm activity and do not demonstrate significant growth during intense storms in comparison with moderate ones. On the other hand, the stronger injection amplitude was calculated during the intense magnetic storm on October 1999.

Detailed investigation of tail and ring current dynamics by the A2000 and G2003 models show that the tail current (as well as other magnetospheric currents) contribution to $D_{s t}$ varies during a magnetic storm. Both models show similar behavior of the $D_{s t}$ sources: the tail current begins to develop earlier than the ring current and starts to decay while the ring current continues to develop. The magnetotail global changes during the magnetic storm are controlled mostly by the solar wind and the IMF, but are accompanied by sharp variations associated with substorms. The G2003 model (Ganushkina et al., 2002; 2004) reproduces the tail current development, which correlates well with the substorm-associated $A E$ index. Clear correlation of the tail current contribution to $D_{s t}$ with substorm activity is also apparent in the results obtained from the A2000 model.

Magnetic field sources contributing to $D_{s t}$ are controlled by different factors originating in the solar wind, as well as in the magnetosphere, which change nonsynchronously, with different time scales and, consequently, determine the complicated dynamics of the $D_{s t}$. Abrupt changes in $D_{s t}$ can be caused either by magnetopause currents in accordance with the IMF and solar wind dynamic pressure pulses, or by tail current variations during substorms. The tail current disruption following substorm onset often influences $D_{s t}$ recovery (Iyemori and Rao, 1996; Kalegaev et al., 2001). Along with the results of Ohtani et al. (2001), the substorm related activity during 02:00-04:00 UT on 26 June 1998 resulted in $D_{s t}$ decay by $30 \mathrm{nT}$ after the substorm onset. Both A2000 and G2003 models reveal such a $D_{s t}$ drop, while the ring current continued to develop. The positive jump from the tail current after substorm maximum is calculated to be about $-40 \mathrm{nT}$ in the A2000 model and about $-50 \mathrm{nT}$ in the G2003 model.

\section{Conclusions}

This study addresses the relation between the ring current and the tail current during storm times. Three different magnetic field models, the paraboloid model A2000 by Alexeev (1978), Alexeev et al. (2001), the event-oriented model G2003 by Ganushkina et al. (2002, 2004), and the T01 model by Tsyganenko (2002a,b) were used to model two storm events. One storm event was moderate with $D_{s t}=-120 \mathrm{nT}$, and another was an intense storm with $D_{s t}=-250 \mathrm{nT}$.

In general, all models showed quite good agreement with in-situ observations. The event-oriented model G2003 represented best the substorm-associated variations of the $B_{z}$ component at and near geosynchronous orbit during both moderate and intense storms. The T01 model provided good agreement between the observed and modelled $B_{x}$ component, but on the other hand, the model $B_{z}$ was significantly more depressed than that observed during the intense storm. Similarly, the A2000 model reproduces well the $B_{x}$ components of the magnetic field measured along the GOES and Polar orbits. 
The A2000, G2003 and T01 models showed that during the moderate storm the tail and ring current contributions are comparable. All three models showed that the tail current develops before the ring current when $D_{s t}$ starts to decrease. During the recovery phase the ring current stays more enhanced than the tail current, according to the A2000 and G2003 model results. The ring current in the T01 model recovers quickly and the tail current remains at an enhanced level almost until the end of the storm recovery.

Similar to the moderate storm, during the intense storm, in all three models the tail current developed first when $D_{s t}$ started to decrease. During the storm maximum the ring current was the dominant contributor to the $D_{s t}$ index in the A2000 and G2003 models. During the early recovery phase the ring current stayed intensified longer than the tail current, becoming comparable to the tail current intensity during the late recovery. In the T01 model the tail current continued to enhance until storm maximum, and gave the largest contribution to the $D_{s t}$ index. During the early recovery phase in the T01 model the tail current contribution decreased rapidly and became comparable to the ring current. Unlike the moderate storm in which the theoretical A2000 and event-oriented G2003 models give a tail current contribution to $D_{s t}$ comparable with the ring current contribution, during the intense storm the ring current becomes the dominant contributor.

The tail current dynamics in the A2000 and G2003 models is correlated well with substorm activity. The tail current enhancement during substorm precedes the $D_{s t}$ recovery, but the ring current continues to develop after the substorm maximum. In agreement with Ohtani et al. (2001), the tail current is responsible for a $D_{s t}$ increase of about $30 \mathrm{nT}$. According to the A2000 and G2003 models, the tail current preintensification level is about -40 to $-50 \mathrm{nT}$.

Magnetic field modelling is a very useful tool not only for the accurate representation of the magnetic field, but also for studies of the evolution of the large-scale current systems. Global models represent well the main features of the magnetospheric magnetic field, but give some discrepancies in representing local magnetic field features. For such cases, event-oriented modelling can be used to improve the accuracy of calculations for specific events.

Acknowledgements. We would like to thank K. Ogilvie and R. Lepping for the use of WIND data in this paper, World Data Center C2 for Geomagnetism, Kyoto, for the provisional $A E, K_{p}$ and $D_{s t}$ indices data. The data were obtained from the Coordinated Data Analysis Web (CDAWeb). GEOTAIL magnetic field data were provided by T. Nagai through DARTS at the Institute of Space and Astronautical Science (ISAS) in Japan. The work of N. Ganushkina was supported by the Academy of Finland. The work of V. Kalegaev was supported by Russian Foundation for Basic Research (Grants 01-07-90117 and 04-05-64396) and INTAS (Grant 03-51-3922).

Topical Editor in chief thanks R. Clauer and another referee for their help in evaluating this paper.

\section{References}

Alekseyev, I. I.: Regular magnetic field in the Earth's magnetosphere, Geomagnetism and Aeronomy, 18, 447-452, 1978.

Alexeev, I. I., Belenkaya, E. S., Kalegaev, V. V., Feldstein, Y. I., and Grafe, A.: Magnetic storms and magnetotail currents, J. Geophys. Res., 101, 7737-7747, 1996.

Alexeev, I. I. and Feldstein, Y. I.: Modelling of geomagnetic field during magnetic storms and comparison with observations, J. Atmos. Sol.-Terr. Phys., 63, 331-340, 2001.

Alexeev I. I., Kalegaev V. V., Belenkaya E. S., Bobrovnikov S. Y., Feldstein Y. I., and Gromova L. I.: Dynamical model of the magnetosphere: case study for 9-12 January 1997, J. Geophys. Res., 106, 25 638-25 693, 2001.

Arykov, A. A. and Maltsev, Yu. P.: Contribution of various sources to the geomagnetic storm field, Geomagnetism and Aeronomy, 33, 67-74, 1993.

Burton, R. K., McPherron, R. L., and Russell, C. T.: An empirical relationship between interplanetary conditions and $D_{s t}$, J. Geophys. Res., 80, 4204-4214, 1975.

Campbell, W. P.: The field levels near midnight at low and equatorial geomagnetic stations, J. Atmos. and Terr. Phys., 35, 1127 1146, 1973.

Dessler, A. J. and Parker, E. N.: Hydromagnetic theory of geomagnetic storms, J. Geophys. Res., 64, 2239-2252, 1959.

Dremukhina, L. A., Feldstein, Y. I., Alexeev, I. I., Kalegaev, V. V., and Greenspan, M.: Structure of the magnetospheric magnetic field during magnetic storms, J. Geophys. Res., 104, $28351-$ $28360,1999$.

Fairfield, D. H., Tsyganenko, N. A., Usmanov, A. V., and Malkov, M. V.: A large magnetospheric magnetic field data base, J. Geophys., Res., 99, 11319-11326, 1994.

Ganushkina, N. Y., Pulkkinen, T. I., Kubyshkina, M. V., Singer, H. J., and Russell, C. T.: Modelling the ring current magnetic field during storms, J. Geophys. Res., 107, 10.1029/2001JA900101, 2002.

Ganushkina, N. Y., Pulkkinen, T. I., Kubyshkina, M. V., Singer, H. J., and Russell, C. T.: Long-term evolution of magnetospheric current systems during storms, Ann. Geophys., 22, 1317-1334, 2004,

SRef-ID: 1432-0576/ag/2004-22-1317.

Greenspan, M. E. and Hamilton, D. C.: A test of the Dessler-ParkerSckopke relation during magnetic storms, J. Geophys. Res., 105, 5419-5430, 2000.

Häkkinen, L., Pulkkinen, T. I., Nevanlinna H., Pirjola R. J., and Tanskanen E. I.: Effect of induced currents on $D_{s t}$ and on magnetic variations at midlatitude stations, J. Geophys. Res., 107 , 10.1029/2001JA900130, 2002.

Hamilton, D. C., Gloeckler, G., Ipavich, F. M., Studemann, W., Wilken, B., and Kremser, G.: Ring current development during the great geomagnetic storm on February 1986, J. Geophys. Res., 93, 14343-14355, 1988.

Iyemory, T. and Rao, D. R. K.: Decay of the $D_{s t}$ field of geomagnetic disturbance after substorm onset and its implication to storm-substorm relation, Ann. Geophys., 14, 608-618, 1996.

Jordanova, V. K., Torbert, R. B., Thorne, R. M., Collin, H. L., Roeder, J. L., and Foster, J. C.: Ring current activity during the early $B_{z}<0$ phase of the January 1997 magnetic cloud, J. Geophys. Res., 104, 24 895-24 914, 1999.

Kalegaev, V. V., Alexeev, I. I., Feldstein, Y. I., Gromova, L. I., Grafe, A., and Greenspan, M.: Tail lobe magnetic flux and dynamics of the $D_{s t}$ disturbance in the course of magnetic storms, 
Geomagnetism and Aeronomie, 38, 10-16, 1998.

Kalegaev V. V., Alexeev, I. I., and Feldstein, Y. I.: The Geotail and Ring Current Dynamics Under Disturbed Conditions, Journal of Atm. and Sol-Terr. Phys., 63, 473-479, 2001.

Kaufmann T. G.: Substorm currents: Growth phase and onset, J. Geophys. Res., 92, 7471-7481 1987.

Liemohn, M. W., Kozyra, J. U., Thomsen, M. F., Roeder, J. L., Lu, G., Borovsky, J. E., and Cayton, T. E.: Dominant role of the asymmetric ring currentin producing the stormtime $D_{s t}^{*}$, J. Geophys. Res., 106, 10 883-10 904, 2001.

Maltsev Y. P., Arykov, A. A., Belova, E. G., Gvozdevsky, B. B., and Safargaleev, V. V.: Magnetic flux redistribution in the storm time magnetosphere, J. Geophys. Res., 101, 7697-7704 1996.

O'Brien, T. P. and McPherron, R. L.: 2000, An empirical phase space analysis of ring current dynamics: Solar wind control of injectionand decay, J. Geophys. Res., 105, 7707-7719, 2000.

Ohtani, S., Nose, M., Rostoker, G., Singer, H., Lui, A. T. Y., and Nakamura, M.: Storm-substorm relationship: Contribution of the tail current to $D_{s t}$, J. Geophys. Res., 106, 21 199-21 209, 2001.

Sckopke, N.: A general relation between the energy of trapped particles and the disturbance field near the Earth. J. Geophys. Res., 71, 3125-3130, 1966.

Shue, J.-H., Chao, J. K., Fu, H. C., Russell, C. T., Song, P., Khurana, K. K., and Singer, H. J.: A new functional form to study the solar wind control of the magnetopause size and shape, J. Geophys. Res., 102, 9497-9512, 1997.
Starkov, G. V.: Planetary morphology of the aurora, In Magnetosphere-Ionosphere Physics, St-Petersburg: Nauka, 8590, 1993.

Sugiura, M. and Kamei, T.: Equatorial $D_{s t}$ index 1957-1986, in IAGA Bull. 40, Edited by A. Berthelier, and M. Menvielle, Int Serv. of Geomagn. Indices Publ. Off., Saint Maur, France, 1991.

Tsyganenko, N. A.: A magnetospheric magnetic field model with a warped tail current sheet, Planet. Space Sci., 37, 5-20, 1989.

Tsyganenko, N. A.: Modeling the Earth's magnetospheric magnetic field confined within a realistic magnetopause, J. Geophys. Res. 100, 5599-5612, 1995.

Tsyganenko, N. A.: A model of the near magnetosphere with a dawn-dusk asymmetry: 1. Mathematical structure, J. Geophys. Res., 107, 10.1029/2001JA0002192001, 2002a.

Tsyganenko, N. A.: A model of the near magnetosphere with a dawn-dusk asymmetry: 2. Parameterization and fitting to observations, J. Geophys. Res., 107, 10.1029/2001JA900120, 2002b.

Tsyganenko, N. A., Singer, H. I., and Kasper, J. C.: Storm-time distortion of the magnetosphere: How severe can it get? J. Geophys. Res., 108, 10.1029/2002JA009808, 2003.

Turner, N. E., Baker, D. N., Pulkkinen,T. I., and McPherron, R. L.: Evaluation of the tail current contribution to $D_{s t}$, J. Geophys. Res., 105, 5431-5440, 2000. 